\title{
O IMPACTO DAS CARACTERÍSTICAS
}

PESSOAIS NA INTENÇÃO DE COMPRA PELA

INTERNET E O PAPEL DE MEDIAÇÃO DA

FAMILIARIDADE E DA ATITUDE ANTE A

COMPRA PELA INTERNET

\section{GABRIEL MARIN GARCIA}

Bacharel em Administração de Empresas pela Escola de Administração da Universidade Federal do Rio Grande do Sul (UFRGS).

Gerente de Projetos da Empresa SAP Labs Latin America.

Avenida SAP, 188, Cristo Rei, São Leopoldo - RS - Brasil - CEP 93022-718

E-mail: garcia.gm@gmail.com

\section{CRISTIANE PIZZUTTII DOS SANTOS}

Doutora em Administração pela Escola de Administração da

Universidade Federal do Rio Grande do Sul (UFGRS).

Professora do Departamento de Ciências Administrativas

da Universidade Federal do Rio Grande do Sul (UFRGS).

Rua Washigton Luis, 855, Centro, Porto Alegre - RS - Brasil - CEP 90010-460

E-mail:cpsantos@ea.ufrgs.br 


\section{RESUMO}

O presente artigo tem como objetivo analisar, dentro do contexto específico de compras na internet, a relação entre características pessoais e intenções de compra, com o propósito de entender o papel da atitude ante a compra pela internet e da familiaridade do consumidor com compras on-line nessa relação. Uma pesquisa descritiva conclusiva foi elaborada visando compreender o efeito de quatro características pessoais: autoeficácia, inovatividade, necessidade de interação social e necessidade de interação sensorial, na familiaridade, na atitude ante a compra pela internet e na intenção de compra on-line. Com base na literatura, um modelo foi construído e testado, usando análise fatorial confirmatória para avaliar o modelo de medidas e análise de equações estruturais para testar as hipóteses deste trabalho. Ao todo, foram aplicados 233 questionários, e os resultados indicaram que existe uma relação significativa entre as características pessoais estudadas e familiaridade com compras na internet. A necessidade de interação social apresentou impacto direto na familiaridade e na atitude, destacando-se como uma característica-chave no processo de compra on-line. Ainda, como resultado, confirmou-se que a familiaridade medeia as relações entre as características pessoais e a atitude ante a compra na internet. Esta última exerce papel central na formação do comportamento de compra, sendo fortemente influenciada pela familiaridade e influenciando diretamente as intenções de compra. Como implicação teórica deste trabalho, tem-se o exame de questões ainda pouco exploradas no campo de conhecimento do comportamento do consumidor on-line, como o papel das características pessoais e da atitude na intenção de compra on-line. Como implicação gerencial, este trabalho proporciona aos administradores um entendimento maior sobre os fatores que influenciam a compra eletrônica.

\section{PALAVRAS-CHAVE}

Intenção de compra; Internet; Características pessoais; Familiaridade; Atitude. 


\section{INTRODUÇÃO}

O comércio eletrônico $\mathrm{B} 2 \mathrm{C}$ (Business to Consumer) vem crescendo rapidamente na última década. Em 20ıo, as empresas de varejo on-line faturaram $\mathrm{R} \$ \mathrm{I} 4,8$ bilhões no Brasil, 40\% a mais que em 2009. O número de brasileiros que realizaram ao menos uma compra na internet em 20 io chegou a 23 milhões - e 5,4 milhões de pessoas compraram pela primeira vez pela internet em 2010 - e estima-se que esse número ultrapasse os 27 milhões em 2011 (EBIT-EMPRESA, 20II). Esses números, ao mesmo tempo que demonstram um crescimento fenomenal da internet como canal de compra no Brasil, também indicam que há ainda um caminho a ser trilhado para que esse canal passe a fazer parte do cotidiano da maioria da população brasileira.

Com o advento da internet e do comércio virtual, as relações consumidorempresa se modificam, assim como o comportamento do cliente. O consumidor possui agora acesso a uma grande quantidade de informações sobre produtos, preços e concorrentes, além da eliminação de barreiras geográficas e de tempo proporcionadas pela internet.

Zhou et al. (2007) afirmam que a proliferação do comércio eletrônico tem estimulado um grande número de pesquisas no intuito de descobrir o que atrai e retém consumidores, tanto em uma visão orientada ao consumidor como em uma visão orientada à tecnologia. A visão orientada aos consumidores foca as crenças dos consumidores sobre compras on-line, visto que tais crenças podem influenciar a seleção do canal de compra. Dentro dessa perspectiva, analisa-se qual a importância de determinados fatores como: características demográficas, psicológicas e cognitivas; percepção do risco e benefícios em relação à compra on-line e motivação e orientação para compra. Por sua vez, a visão orientada à tecnologia tenta explicar e prever o comportamento do consumidor virtual por meio da análise técnica das especificações de uma loja virtual, que incluem interface do usuário, conteúdo e design do website e usabilidade do sistema.

Neste estudo, optou-se por focar a perspectiva do consumidor, especificamente o efeito das características pessoais no comportamento de compra. As características pessoais definem nossa personalidade, ou seja, os padrões consistentes e previsíveis que direcionam nosso estilo de vida e o que fazemos. Elas são particularmente importantes no estágio de reconhecimento de necessidades e na tomada de decisão de compra (ENGEL; BLACKWELL; MINIARD, 2005).

Segundo os estudos de Dabholkar (I996) e Dabholkar e Bagozzi (2002), torna-se relevante estudar as características dos consumidores virtuais, baseando-se não apenas em critérios tradicionais de segmentação, mas também em 
critérios ligados a variáveis da personalidade, especialmente aquelas ligadas às atitudes em relação ao uso e conforto com a tecnologia. As características demográficas, apesar de serem relevantes, não são críticas para entender por que consumidores utilizam tecnologia, e os estudos psicográficos oferecem bons insights para profissionais de marketing, mas não vão longe o bastante para descobrir quais as reais motivações dos consumidores. As variações no comportamento do consumidor baseiam-se muito mais nas características pessoais dos consumidores do que em fatores demográficos e psicográficos, por que as variações na personalidade estão no ponto central da formação da atitude e intenção de comportamento.

Os efeitos das características pessoais na compra eletrônica e sua relação com a atitude ainda são um assunto em maturação. Por tal razão, não há uma definição clara de quais características pessoais têm papel relevante na decisão de compra e de como elas influenciam esse processo. Todavia, algumas características pessoais, como autoeficácia, necessidade de interação social, necessidade de interação sensorial e inovatividade se destacam, sendo já investigadas em diversos estudos como os de Dabholkar (1996), Dabholkar e Bagozzi (2002), Clarke III e Flaherty (2005) e Peck e Childers (2003), por terem relação com o uso de tecnologia. Este trabalho visa ampliar o estudo dessas características pessoais, vinculando essas características ao processo de compra eletrônica.

Com base na literatura sobre o tema (LAROSE; EASTIN, 2000; BELLMAN; LOHSE; JOHNSON, I999; BROWN; POPE; VOGES, 2003), além da influência na intenção de compra, as características pessoais teriam impacto em duas importantes variáveis: familiaridade com compras na internet e atitude ante a compra pela internet. Enquanto a ligação entre características pessoais e atitude tem sido estudada por alguns autores, como Dabholkar e Bagozzi (2002), Peck e Childers (2003) e Agarwal e Prasad (I998), o impacto das características pessoais na familiaridade tem sido fortemente ignorado, com exceção de LaRose e Eastin (2000) que encontraram que o nível de autoeficácia do indíviduo influencia diretamente a familiaridade com a tecnologia. Familiaridade tem sido investigada, basicamente, como antecedente de variáveis como confiança (YOON, 2003; SANTOS; FERNANDES, 2007) e atitudes (BELLMAN; LOHSE; JOHNSON, I999; BROWN; POPE; VOGES, 2003). Dessa forma, este trabalho se propõe a analisar, dentro do contexto específico de compras na internet, a relação entre características pessoais e intenções de compra, com o propósito de entender o papel de mediação da atitude ante a compra na internet e da familiaridade do consumidor com compras on-line nessa relação. 


\section{REFERENCIAL TEÓRICO E HIPÓTESES DE PESQUISA}

\subsection{CARACTERÍSTICAS PESSOAIS}

Pelo fato de o comportamento dos consumidores estar ligado aos seus valores individuais, torna-se importante estudar os traços da personalidade. A personalidade é definida como respostas consistentes a estímulos ambientais. Dessa forma, a personalidade do indivíduo proporciona experiências e comportamentos apresentados de forma ordenada e coerente. O padrão particular de organização de cada pessoa a torna única, e nele a personalidade é um fator determinante, proporcionando consistência de respostas baseadas em características psicológicas internas (ENGEL; BLACKWELL; MINIARD, 2005).

As características pessoais dos consumidores têm um importante papel na adoção de produtos tecnológicos (AGARWAL; PRASAD, I998). Dabholkar e Bagozzi (2002) afirmam que questões relacionadas a quais características pessoais determinam interesse em serviços tecnológicos estão se tornando crescentemente relevantes. A seguir, abordar-se-ão quatro características pessoais importantes e seus efeitos na atitude, familiaridade e intenção de compra.

\subsection{AUTOEFICÁCIA}

Define-se autoeficácia como a avaliação do indíviduo sobre sua habilidade de executar algum comportamento (DABHOLKAR; BAGOZZI, 2002). As pessoas com crenças robustas sobre sua eficácia para alcançar determinado desempenho tendem a persistir em ultrapassar obstáculos e são menos propensas a exibir ansiedade quando defrontadas com ameaças dos ambientes (BANDURA, I999; STAJKOVIC; LUTHANS, I998; CAPRARA; CERVONE, 2000). Autoeficácia seria, então, um dos grandes fatores que determinam a motivação intrínsica (DAVIS; BAGOZZI; WARSHAW, I989).

Embora a autoeficácia tenha sido originalmente concebida como variável relacionada especificamente a uma determinada tarefa, alguns pesquisadores têm ressaltado a existência da autoeficácia generalizada, a qual prevê o comportamento individual entre várias situações e circunstâncias (TIPTON; WORTHINGTON, I984; CHEN; GULLY; EDEN, 200I; YEO; NEAL, 2006).

Segundo Bandura (I982), a autoeficácia funciona como um moderador cognitivo de ação. Nesse sentido, as pessoas que acreditam possuir o conhecimento necessário e as habilidades para realizar determinada tarefa se sentem mais confortáveis em realizá-la. Estendendo essa lógica para o comportamento de compra 
via internet, consumidores com maior autoeficácia teriam maior confiança em suas habilidades e se sentiriam mais confortáveis para usar tecnologia e fazer compras on-line. Sendo assim, formulam-se as primeiras hipóteses deste trabalho:

- Hr: Consumidores com maior autoeficácia terão uma atitude mais positiva ante a compra pela internet.

- H2: Consumidores com maior autoeficácia serão mais inclinados a usar a internet como canal de compras.

\subsection{NECESSIDADE DE INTERAÇÃO SOCIAL}

A necessidade de contato pessoal na entrega de serviços é extremamente importante para alguns consumidores (SIMON; USUNIER, 2007). Por sua vez, Meuter et al. (2000) afirmam que evitar contato pessoal na prestação de serviço pode ser fonte de satisfação e, por tal razão, influenciar positivamente o uso de autosserviços tecnológicos. Dabholkar (I996) e Dabholkar e Bagozzi (2002) definem necessidade de interação social como "a importância da interação humana na prestação de serviços” e dizem que essa caracaterística tem um papel relevante no uso de autosserviços tecnológicos. Se os consumidores tiverem uma alta necessidade de interação, eles serão relutantes em usar o autosserviço. Entretanto, se houver uma baixa necessidade de interação, eles darão preferência ao uso do autosserviço. Propõe-se então que:

- H3: Consumidores com maior necessidade de interação social terão uma atitude mais negativa ante a compra pela internet.

- $\quad \mathrm{H}_{4}$ : Consumidores com maior necessidade de interação social serão menos inclinados a usar a internet como canal de compras.

\subsection{NECESSIDADE DE INTERAÇÃO SENSORIAL}

Enquanto atributos sensoriais do produto podem ter diferentes influências na usabilidade da internet como um canal de vendas, muitos pesquisadores examinaram a necessidade de tocar o produto como uma característica individual de cada consumidor. Desde os primórdios do comércio eletrônico, pesquisadores identificaram a relação negativa entre compras pela internet e a importância dada pelos consumidores a uma experiência sensorial com os produtos antes da compra (FALK; TALARZYK; WIDING II, I994). Segundo Peck e Childers (2003), na área de marketing, a necessidade de interação sensorial afeta tanto a atitude em relação ao produto como o próprio comportamento de compra. A 
atitude em relação ao produto pode depender diretamente da possibilidade de o comprador tocar o produto e experienciar um retorno sensorial. A necessidade de sentir e tocar o produto antes da compra pode ser o discriminador entre os adotantes e não adotantes da internet como canal de compra. Esse fator irá afetar diretamente a probabilidade de o consumidor vir a comprar na internet. Com base nisso, pode-se propor que:

- $\quad \mathrm{H}_{5}$ : Consumidores com maior necessidade de interação sensorial terão uma atitude mais negativa ante a compra pela internet.

- H6: Consumidores com maior necessidade de interação sensorial serão menos inclinados a usar a internet como canal de compras.

\subsection{INOVATIVIDADE}

Inovatividade mede em que velocidade e em qual extensão um indíviduo adota inovações está relacionada com compras pela internet, o que pode ser tratado como um comportamente inovador em relação à compra em lojas físicas (ZHOU et al., 2007). A inovatividade ou busca por novidades também é definida como um desejo de procurar um novo estímulo (HIRSCHMAN, I980). É correntemente aceito que aspectos da inovatividade poderão influenciar a atitude em relação a produtos tecnológicos, isto é, quanto maior for a inovatividade do sujeito, mais positiva será a atitude em relação ao uso da tecnologia (DABHOLKAR; BAGOZZI, 2002). Com base na literatura consultada, propõe-se que:

- H7: Consumidores com maior inovatividade terão uma atitude mais positiva ante a compra na internet.

- H8: Consumidores com maior inovatividade serão mais inclinados a usar a internet como canal de compras.

\subsection{ATITUDE ANTE A COMPRA PELA INTERNET}

Segundo Sheth, Mittal e Mewman (200I), a atitude do consumidor é um composto de crenças, sentimentos e intenções de comportamento em direção a algum produto ou objeto. Para Engel, Blackwell e Miniard (2005), a atitude refere-se a gostos e desgostos do indíviduo que determinam suas intenções. A atitude em relação ao comércio eletrônico é de fundamental importância no momento da decisão da compra on-line. Especificamente, a utilidade e o valor que os consumidores esperam obter da experiência de compras estão diretamente relacionados com a probabilidade de usar a internet para compra. A atitude pode ser 
considerada um dos conceitos mais importantes no estudo do comportamento do consumidor, em razão do seu papel verificado na literatura de determinante direto do comportamento (SHETH; MITTAL; NEWMAN, 200I). Assim, uma vez que se sabem quais atitudes são relevantes para um estímulo particular, é possível prever o comportamento baseado nesse estímulo.

Atitude ante a compra on-line é, de fato, um importante fator que diferenciará os compradores virtuais dos não compradores (GOLDSMITH; BRIDGES, 2000). Os primeiros valorizam conveniência, seleção e tempo como benefícios de comprar on-line, enquanto os segundos estão mais preocupados com segurança, perda da privacidade e não recebimento das mercadorias. Atitude em relação à tecnologia, particularmente à internet, influencia a propensão de fazer compras on-line, e a percepção de utilidade do comércio eletrônico (CLARKE III; FLAHERTY, 2005). Além disso, atitude em relação à tecnologia também influencia a percepção dos consumidores quanto aos riscos associados com a compra on-line (LEE; TAN, 2005).

Dabholkar (1996) criou um modelo para estudar a atitude dos consumidores em relação à intenção de usar autosserviços baseados em tecnologia. O modelo de influência geral (overall affect model) é baseado em uma visão de um processo de influência na decisão de compra, no qual consumidores já teriam predisposições para formar expectativas da qualidade do serviços baseados em tecnologia exclusivamente. A maioria das pessoas provalvelmente já foi exposta, em algum nível, a produtos/serviços tecnológicos e consequentemente já formou algum tipo de atitude em relação a esses produtos, variando de muito favorável a pouco favorável. Em situações novas, mas similares, a predisposição em relação à tecnologia tem influência na intenção de usar novos produtos ou serviços, como a internet, porque os consumidores se sentem mais confortáveis na interação com a tecnologia. Propõe-se então, que:

- H9: Quanto mais positiva for a atitude do consumidor ante a compra pela internet, mais inclinado ele estará ao uso dessa tecnologia como canal de compras.

\subsection{FAMILIARIDADE DO CONSUMIDOR}

O nível de expertise com o veículo da internet, tanto como um repositório de informações quanto como uma ferramenta de compra, é considerado por muitos especialistas como o fator-chave para prever compras on-line (BELLMAN; LOHSE; JOHNSON, I999; BROWN; POPE; VOGES, 2003; GOLDSMITH; BRIDGES, 2000). Para Bruner II e Kumar (2000), o principal determinante da 
reação do consumidor em relação a sites de internet e propagandas na web é a experiência com a internet. Para Brown, Pope e Voges (2003), a frequência de compras on-line está positivamente relacionada com a tendência de comprar on-line e negativamente relacionada com a probabilidade de abortar uma transação. Já para Novak, Hoffman e Yung (2000), usuários mais antigos ou experientes com o uso da web têm maiores habilidades para achar o que estão procurando, sabem escolher os melhores meios para efetuar o pagamento e sabem identificar a loja virtual que oferece maior segurança na transmissão dos dados. Além disso, eles também conseguem concluir a compra com maior rapidez e facilidade porque já conhecem o processo, o que lhes garante uma confiança na hora de concluir a compra, o que um comprador iniciante não teria. Assim, quanto mais tempo os usuários gastam no ambiente on-line, mais tranquilos e familiarizados eles parecem se tornar com relação às características e aos serviços da internet, ao mesmo tempo que ficam mais dispostos a explorá-los (ZHAO, 2006).

O grau de familiaridade com compras na internet parece ser afetado pelas características pessoais. De fato, LaRose e Eastin (2000) constataram que alta autoeficácia é essencial para que usuários iniciantes superem o medo de usar a internet. Em seus estudos, eles observaram que a autoeficácia está positivamente relacionada com a experiência na internet. Estendendo esse raciociocínio para as demais características pessoais estudadas neste trabalho e mantendo as mesmas relações encontradas na literatura, poder-se-ia dizer que quanto maiores as necessidades de interação social e sensorial do sujeito, menor seria sua experiência ou familiaridade com compras on-line, pois naturalmente o consumidor buscaria ambientes nos quais pudesse suprir essa alta necesssidade de interação, o que não é priorizado, para não dizer ausente, pela internet. O contrário aconteceria com a inovatividade. Sujeitos com alto nível de inovatividade provavelmente utilizariam com maior facilidade o meio eletrônico para compras, por este ser inovador se comparado à compra em lojas físicas. Assim, sugere-se a seguinte hipótese:

- Hio: Consumidores com maior autoeficácia (Io A), maior inovatividade $\left(\mathrm{I}_{\mathrm{B}}\right)$, menor necessidade de interação social ( $\mathrm{Io}_{\mathrm{C}}$ ) e menor necessidade de interação sensorial ( $\mathrm{IO}_{\mathrm{D}}$ ) terão maior familiaridade com compras pela internet.

Além disso, as experiências anteriores são um componente do estágio de conhecimento e ajuda na formação de atitude ante a adoção de uma inovação (ROGERS, I995). Yoh et al. (2003) afirmam que os consumidores tendem, por meio da familiaridade, a acumular conhecimento e fortes crenças sobre o siste- 
ma - internet -, o que aumenta suas atitudes gerais ante ela. Já Santos e Fernandes (2007) constatam que, no comércio virtual, experiências anteriores com compras on-line impactam na confiança do consumidor no meio eletrônico e pela confiança influenciam a intenção de recompra. Assim, se a experiência com compras na internet for ruim, os consumidores poderão desenvolver um sentimento de desconfiança generalizado ante a web, diminuindo sua intenção de recompra via internet. Entende-se então que:

- Hıг: Quanto maior for a familiaridade do consumidor com compras pela internet, mais positiva será sua atitude ante a compra por esse sistema.

- Hi2: Quanto maior a familiaridade do consumidor com compras pela internet, mais inclinado ele estará para utilizar a internet como canal de compras.

Com base na fundamentação teórica e nas hipóteses estabelecidas, apresentase na Figura I o modelo teórico a ser investigado.

\section{FIGURA I}

MODELO TEÓRICO SOBRE O IMPACTO DAS CARACTERÍSTICAS PESSOAIS NA INTENÇÃO DE COMPRA PELA INTERNET E A MEDIAÇÃO DA FAMILIARIDADE E DAS ATITUDES

Características pessoais

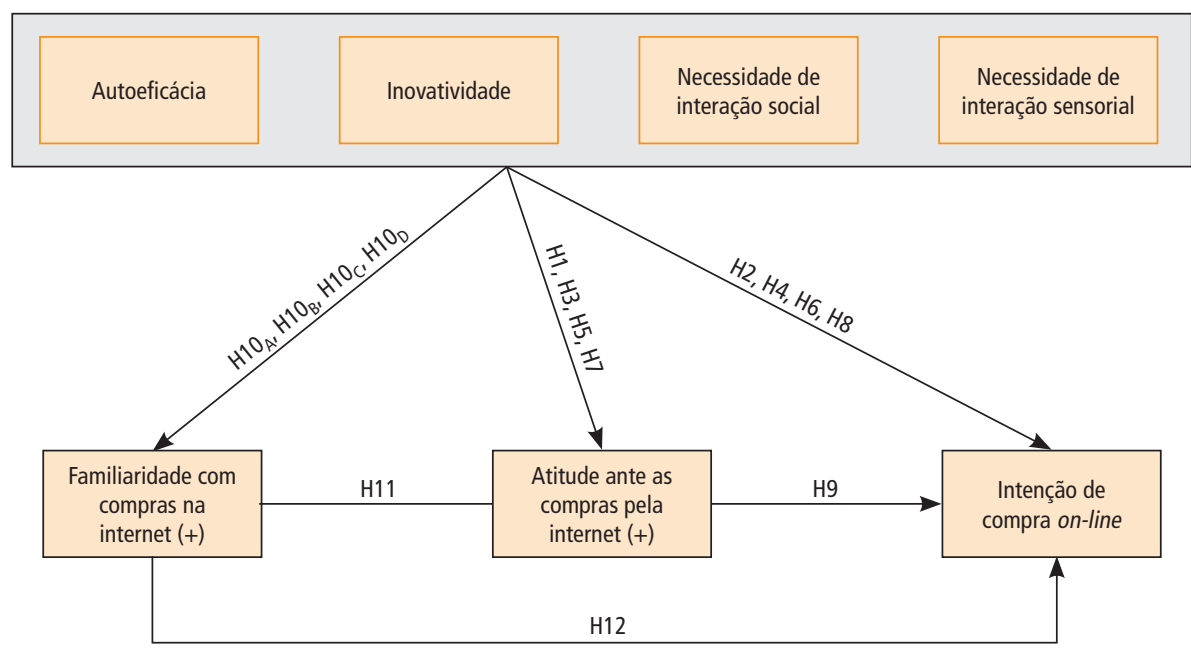

Fonte: Elaborada pelos autores. 


\section{PROCEDIMENTO METOdOLóGICO}

Foi realizado um estudo descritivo (survey) com 233 estudantes de pós-graduação de cinco instituições de ensino superior do Rio Grande do Sul: UFRGS, Unisinos, Feevale, Faculdade São Judas Tadeu e I-UMA (Instituto Universal de Marketing em Agribusiness). Essa amostra é não probabilística e foi escolhida em razão da facilidade de acesso por parte do pesquisador e pela maior heterogeneidade desse público, se comparado a estudantes de graduação. Além disso, acredita-se que grande parte dos alunos de especialização, por estarem no mercado de trabalho e terem certo poder aquisitivo (todos os cursos são pagos), utiliza a internet diariamente, até mesmo como canal de compras.

Com base nos dados obtidos nessa etapa e na revisão da literatura, elaborou-se um questionário estruturado que foi utilizado na etapa descritiva conclusiva.

\subsection{OPERACIONALIZAÇÃO DAS VARIÁVEIS}

Para cada uma das variáveis exploradas neste trabalho, procurou-se identificar um modelo de instrumento que pudesse ser adaptado ou aplicado na íntegra e que possuísse alto grau de confiabilidade; avaliado por meio do alfa de Cronbach do instrumento completo, quando disponibilizado pelos autores, ou das cargas fatoriais dos itens. Todas as escalas foram traduzidas para o português por tradução reversa. A tradução para o português foi conduzida por um dos autores com domínio da língua inglesa, seguida de nova tradução para a língua original feita pelo outro autor, bilíngue. Na sequência, um professor de inglês bilíngue analisou o documento traduzido e a versão original e confirmou que a tradução estava bem feita. Buscou-se utilizar escalas que haviam sido aplicadas no contexto da internet, dessa forma mudanças mínimas foram necessárias.

Todas as questões deste trabalho foram medidas por uma escala Likert de 5 pontos, sendo I para discordo totalmente e 5 para concordo totalmente. No Quadro I, estão as escalas utilizadas e as respectivas fontes. Os itens usados em cada escala são apresentados na Tabela I. 


\section{QUADRO I}

\section{INSTRUMENTOS DE MEDIDA}

\begin{tabular}{|c|c|c|}
\hline $\begin{array}{l}\text { CARACTERÍSTICAS } \\
\text { PESSOAIS }\end{array}$ & $\begin{array}{l}\text { FONTE DO INSTRUMENTO DE } \\
\text { MEDIDA }\end{array}$ & $\begin{array}{l}\text { ITENS UTILIZADOS NAS ESCALAS E CONSISTÊNCIA } \\
\text { INTERNA DO INSTRUMENTO ORIGINAL }\end{array}$ \\
\hline Autoeficácia & $\begin{array}{l}\text { Kulviwat, Bruner II e } \\
\text { Neelankavil (2005) }\end{array}$ & $\begin{array}{l}\text { Aplicação do instrumento completo } \\
\text { (4 itens). Alfa de Cronbach =0,91. }\end{array}$ \\
\hline Inovatividade & $\begin{array}{l}\text { Escala de busca por } \\
\text { novidade adaptada de } \\
\text { Mehrabian e Russell (1974) } \\
\text { por Dabholkar e Bagozzi } \\
\text { (2002) }\end{array}$ & $\begin{array}{l}\text { Aplicação de instrumento com } 3 \text { questões. } \\
\text { Alfa de Cronbach da escala original = 0,72. }\end{array}$ \\
\hline $\begin{array}{l}\text { Necessidade de } \\
\text { interação social }\end{array}$ & $\begin{array}{l}\text { Escala de necessidade de } \\
\text { interação de Dabholkar } \\
\text { (1996) }\end{array}$ & $\begin{array}{l}\text { Aplicação do instrumento completo de } \\
\text { Dabholkar, (4 questões). } \\
\text { Alfa de Cronbach }=0,83 .\end{array}$ \\
\hline $\begin{array}{l}\text { Necessidade } \\
\text { de interação } \\
\text { sensorial }\end{array}$ & $\begin{array}{l}\text { Need for touch scale (NFT), } \\
\text { de Peck e Childers (2003) }\end{array}$ & $\begin{array}{l}\text { Aplicação de } 6 \text { questões da escala NFT, } \\
\text { composto por } 12 \text { questões. Não foi possível } \\
\text { obter as cargas fatoriais dos itens, mas, em } \\
\text { contato com a pesquisadora Joan Peck, ela } \\
\text { garantiu que todas essas questões possuem } \\
\text { cargas fatoriais altas. }\end{array}$ \\
\hline $\begin{array}{l}\text { Atitude ante } \\
\text { a compra pela } \\
\text { internet }\end{array}$ & Kim, Lee e Kim (2004) & $\begin{array}{l}\text { Duas questões de atitude em relação à } \\
\text { compra pela internet (cargas fatoriais acima } \\
\text { de } 0,90 \text { ). }\end{array}$ \\
\hline $\begin{array}{l}\text { Familiaridade } \\
\text { com compras } \\
\text { na internet }\end{array}$ & Bart et al. (2005) & $\begin{array}{l}\text { Aplicação de instrumento com } 3 \text { itens. Alfa } \\
\text { de Cronbach da escala original = 0,93. }\end{array}$ \\
\hline $\begin{array}{l}\text { Intenção de } \\
\text { compra }\end{array}$ & Hausman e Siekpe (2009) & $\begin{array}{l}\text { Aplicação de instrumento com } 3 \text { questões* } \\
\text { Cargas fatoriais acima de } 0,90 \text {. }\end{array}$ \\
\hline
\end{tabular}

* O instrumento original possuía 4 itens, porém dois deles eram muito similares, o que, segundo Netemeyer, Bearden e Sharma (2003), aumentaria a confiabilidade do instrumento artificialmente. Por esse motivo, optou-se pela retirada de um desses itens.

Fonte: Elaborado pelos autores. 


\subsection{PROCEDIMENTOS DE AMOSTRAGEM E COLETA DE DADOS}

Um pré-teste do questionário foi realizado em uma turma de pós-graduandos em Psicologia da Universidade Federal do Rio Grande do Sul (UFRGS), com aplicação de dez questionários. A partir desse pré-teste, recolheram-se sugestões de melhorias na linguagem, para tornar mais claro o entendimento das questões. Nenhuma alteração significativa teve que ser feita.

Após o pré-teste, iniciou-se a aplicação dos questionários nas diversas instituições já citadas, obtendo-se 235 unidades amostrais, das quais 233 foram consideradas válidas. Para realizar essa coleta, foram feitos contatos com os coordenadores ou professores de pós-graduação dessas universidades, e posteriormente agendada a coleta, realizada pelos próprios pesquisadores.

\section{RESULTADOS}

Os resultados deste estudo serão discutidos na seguinte ordem: primeiramente será apresentada a caracterização da amostra. A seguir, o modelo de medidas será investigado por meio da análise fatorial confirmatória, para então serem analisadas as hipóteses do trabalho por meio da análise de equações estruturais (software EQS II.o).

\subsection{CARACTERIZAÇÃO DA AMOSTRA}

Em relação aos dados demográficos, $63,8 \%$ da amostra foram representados por mulheres e a faixa etária com maior número de respondentes foi a de $20 \mathrm{a}$ 30 anos, com $53 \%$. Isso se deve ao fato de a amostra ser de estudantes de pósgraduação, que geralmente se encontram nessa faixa etária. A maior parte dos respondentes se enquadra na faixa de renda entre $\mathrm{R} \$ 3.000$ e $\mathrm{R} \$ 7.000$, representando $52 \%$ da amostra.

A maioria das pessoas (63\%) afirmou ter algum tipo de experiência com compras na internet e cerca de $21,70 \%$ se consideraram muito experientes. A maior parte delas (34\%) adquire de um a três itens a cada seis meses e gasta, em média, até $\mathrm{R} \$ 200,00$ (cerca de 40\%).

\subsection{VALIDADE E CONFIABILIDADE DAS MEDIDAS}

A partir das recomendações de vários autores (HAIR et al., I998; CHURCHILL, I999), a validade desse modelo será suportada, basicamente, se: I. o 
modelo de medidas se ajustar aos dados razoavelmente bem, isto é, dentro dos índices de ajustamento considerados satisfatórios; 2 . as cargas fatoriais dos indicadores nos fatores correspondentes forem grandes e significativas; 3 . indicadores de um mesmo construto produzirem índices de confiabilidade superiores a 0,70 e variância extraída acima de 0,$50 ; 4$. as correlações entre os construtos produzirem evidência de validade discriminante.

Por meio da análise fatorial confirmatória, foram encontradas as cargas fatoriais dos itens em cada construto (Tabela I). As cargas fatoriais foram altas, oscilando entre 0,69 (inovatividade) e 0,95 (atitude ante a compra na internet). Ainda, visando fornecer maior ajustamento ao modelo proposto, itens que apresentaram cargas fatoriais muito baixas foram removidos. Ao todo, removeramse um item do instrumento de autoeficácia e dois do instrumento de necessidade de interação sensorial. Os coeficientes de confiabilidade foram calculados de maneira a garantir a confiabilidade do instrumento, assim como a variância extraída. No geral, o instrumento encontrou índices de confiabilidade satisfatórios, todos acima de 0,70 . A autoeficácia apresentou um índice de confiabilidade de 0,75 . Outros índices encontrados foram: necessidade de interação social $(0,79)$, necessidade de interação sensorial $(0,90)$, inovatividade $(0,80)$, familiaridade com compras na internet $(0,90)$, atitude ante a compra na internet $(0,89)$ e intenções de compra $(0,89)$. Quanto à variância extraída, com exceção da autoeficácia $(0,49)$, todos os construtos tiveram a variância extraída igual ou acima de 0,50 .

Evidência de validade discriminante, que é particularmente importante quando construtos são similares por natureza, foi encontrada por meio dos níveis de correlação existentes entre os construtos. Correlações acima de o,80 indicariam falta de validade discriminante, isto é, construtos estariam medindo o mesmo fenômeno. Todos os construtos foram encontrados distintos uns dos outros. Conforme pode ser visto na Tabela I, a maior correlação foi entre atitude e familiaridade com compras na internet $(0,79)$.

Para testar a multicolinearidade, que se refere a uma situação na qual variáveis preditivas do modelo de regressão são fortemente correlacionadas, foi calculado o índice de variance inflation factor (VIF), que quantifica a severidade da multicolinearidade. Um VIF acima de 5 indica problemas nesse aspecto (O’BRIEN, 2007). Conforme se pode observar na Tabela I, os índices VIF ficaram abaixo desse nível, sendo o maior deles relacionado à variável atitude ante a compra na internet. 


\section{TABELA I}

MATRIZ DE CORRELAÇÃO ENTRE AS VARIÁVEIS LATENTES E VIF

\begin{tabular}{lcccccccc}
\hline VARIÁVEIS & VIF & 1 & 2 & 3 & 4 & 5 & 6 & 7 \\
\hline 1. Familiaridade & 3,605 & 0,74 & & & & & \\
\hline 2. Atitude & 4,143 & 0,83 & 0,79 & & & & & \\
\hline $\begin{array}{l}\text { 3. Intenção de } \\
\text { compra }\end{array}$ & 2,575 & 0,70 & 0,76 & 0,73 & & & & \\
\hline $\begin{array}{l}\text { 4. Autoeficácia } \\
\text { 5. Necessidade de } \\
\text { int. social }\end{array}$ & 1,225 & 0,38 & 0,36 & 0,33 & 0,50 & & \\
\hline $\begin{array}{l}\text { 6. Necessidade de } \\
\text { int. sensorial }\end{array}$ & 1,329 & $-0,18$ & $-0,07$ & $-0,18$ & $-0,04$ & 0,42 & 0,75 & \\
\hline \begin{tabular}{l} 
7. Inovatividade \\
\hline
\end{tabular} & 1,218 & 0,20 & 0,16 & 0,10 & 0,22 & 0,15 & 0,24 & 0,57 \\
\hline
\end{tabular}

Fonte: Elaborada pelos autores.

A Tabela 2 apresenta cargas fatoriais, índices de confiabilidade e variância extraída das medidas.

\section{TABELA 2}

CARGAS FATORIAIS, CONFIABILIDADE, VARIÂNCIA EXTRAÍDA E MÉDIA DAS MEDIDAS

\begin{tabular}{lcccc}
\hline & $\begin{array}{c}\text { CARGAS } \\
\text { FATORIAIS }\end{array}$ & $\begin{array}{c}\text { ÍNDICES DE } \\
\text { CONFIABILIDADE }\end{array}$ & $\begin{array}{c}\text { VARIÂNCIA } \\
\text { EXTRAÍDA }\end{array}$ & $\begin{array}{c}\text { MÉDIA } \\
\text { (DESVIO } \\
\text { PADRÃO) }\end{array}$ \\
\hline Autoeficácia & & 0,75 & 0,50 & $4,41(0,68)$ \\
\hline $\begin{array}{l}\text { 1. AE 1 (Me sinto apto a usar a internet por } \\
\text { conta própria) }\end{array}$ & 0,67 & & \\
\hline $\begin{array}{l}\text { 2. AE 2 (Tenho tempo necessário para fazer } \\
\text { a internet útil para mim) }\end{array}$ & 0,60 & & \\
\hline $\begin{array}{l}\text { 3. AE 3 (Tenho o conhecimento e } \\
\text { habilidades necessárias para usar a } \\
\text { internet) }\end{array}$ & $0,8,31)$ & & \\
\hline
\end{tabular}




\section{TABela 2 (CONTINUAÇÃo)}

\section{CARGAS FATORIAIS, CONFIABILIDADE, VARIÂNCIA EXTRAÍDA E MÉDIA DAS MEDIDAS}

\begin{tabular}{|c|c|c|c|c|}
\hline & $\begin{array}{l}\text { CARGAS } \\
\text { FATORIAIS }\end{array}$ & $\begin{array}{l}\text { ÍNDICES DE } \\
\text { CONFIABILIDADE }\end{array}$ & $\begin{array}{l}\text { VARIÂNCIA } \\
\text { EXTRAÍDA }\end{array}$ & $\begin{array}{l}\text { MÉDIA } \\
\text { (DESVIO } \\
\text { PADRÃO) }\end{array}$ \\
\hline Necessidade de interação social & & 0,79 & 0,49 & $3,78(0,87)$ \\
\hline $\begin{array}{l}\text { 1. NI } 1 \text { (O contato humano torna o } \\
\text { processo de compra mais agradável para } \\
\text { o consumidor) }\end{array}$ & 0,70 & & & \\
\hline $\begin{array}{l}\text { 2. NI } 2 \text { (Gosto de interagir com a pessoa } \\
\text { que vende o produto) }\end{array}$ & $\begin{array}{c}0,81 \\
(9,75)\end{array}$ & & & \\
\hline $\begin{array}{l}\text { 3. NI } 3 \text { (A atenção pessoal do atendente é } \\
\text { muito importante) }\end{array}$ & $\begin{array}{l}0,65 \\
(8,31)\end{array}$ & & & \\
\hline $\begin{array}{l}\text { 4. NI } 4 \text { (Eu me incomodo de usar uma } \\
\text { máquina quando posso falar diretamente } \\
\text { com o atendente da loja) }\end{array}$ & $\begin{array}{c}0,64 \\
(8,26)\end{array}$ & & & \\
\hline Necessidade de interação sensorial & & 0,90 & 0,75 & $3,14(1,18)$ \\
\hline $\begin{array}{l}\text { 1. NIS } 1 \text { (Confio mais nos produtos que eu } \\
\text { posso tocar antes de fazer a compra) }\end{array}$ & 0,83 & & & \\
\hline $\begin{array}{l}\text { 2. NIS } 2 \text { (Eu me sinto mais confortável } \\
\text { comprando um produto que posso } \\
\text { examinar) }\end{array}$ & $\begin{array}{c}0,94 \\
(16,34)\end{array}$ & & & \\
\hline $\begin{array}{l}\text { 3. NIS } 3 \text { (Quando eu visito lojas, gosto de } \\
\text { tocar muitos produtos) }\end{array}$ & $\begin{array}{c}0,82 \\
(14,51)\end{array}$ & & & \\
\hline Inovatividade & & 0,80 & 0,57 & $3,86(0,79)$ \\
\hline $\begin{array}{l}\text { 1. INO } 1 \text { (Estou sempre buscando novas } \\
\text { ideias e experiências) }\end{array}$ & 0,65 & & & \\
\hline $\begin{array}{l}\text { 2. INO } 2 \text { (Gosto de continuamente mudar } \\
\text { minhas atividades) }\end{array}$ & $\begin{array}{c}0,77 \\
(8,66)\end{array}$ & & & \\
\hline $\begin{array}{l}\text { 3. INO } 3 \text { (Gosto de experimentar novidades } \\
\text { e alterar minha rotina diária) }\end{array}$ & $\begin{array}{c}0,83 \\
(8,67)\end{array}$ & & & \\
\hline
\end{tabular}




\section{TABELA 2 (CONCLUSÃO)}

\section{CARGAS FATORIAIS, CONFIABILIDADE, VARIÂNCIA EXTRAÍDA E MÉDIA DAS MEDIDAS}

\begin{tabular}{|c|c|c|c|c|}
\hline & $\begin{array}{l}\text { CARGAS } \\
\text { FATORIAIS }\end{array}$ & $\begin{array}{l}\text { ÍNDICES DE } \\
\text { CONFIABILIDADE }\end{array}$ & $\begin{array}{l}\text { VARIÂNCIA } \\
\text { EXTRAÍDA }\end{array}$ & $\begin{array}{c}\text { MÉDIA } \\
\text { (DESVIO } \\
\text { PADRÃO) }\end{array}$ \\
\hline Familiaridade com compras na internet & & 0,90 & 0,74 & $2,77(1,23)$ \\
\hline $\begin{array}{l}\text { 1. EXP } 1 \text { (Tenho familiaridade com compras } \\
\text { pela internet) }\end{array}$ & 0,89 & & & \\
\hline $\begin{array}{l}\text { 2. EXP } 2 \text { (Estou acostumado(a) a usar a } \\
\text { internet como canal de compras) }\end{array}$ & $\begin{array}{c}0,96 \\
(22,28)\end{array}$ & & & \\
\hline $\begin{array}{l}\text { 3. EXP } 3 \text { (Tenho boa experiência em } \\
\text { compras na internet) }\end{array}$ & $\begin{array}{c}0,72 \\
(13,12)\end{array}$ & & & \\
\hline Atitude ante a compra pela internet & & 0,89 & 0,80 & $3,24(1,20)$ \\
\hline 1. Al 1 (Gosto de comprar na internet) & 0,96 & & & \\
\hline $\begin{array}{l}\text { 2. Al } 2 \text { (Lojas on-line são bons lugares para } \\
\text { fazer compra) }\end{array}$ & $\begin{array}{c}0,82 \\
(16,81)\end{array}$ & & & \\
\hline Intenções de compra & & 0,89 & 0,73 & $3,92(0,99)$ \\
\hline $\begin{array}{l}\text { 1. IC } 1 \text { (Definitivamente irei comprar na } \\
\text { internet) }\end{array}$ & 0,76 & & & \\
\hline $\begin{array}{l}\text { 2. IC } 2 \text { (Pretendo comprar na internet no } \\
\text { futuro próximo) }\end{array}$ & $\begin{array}{c}0,95 \\
(14,49)\end{array}$ & & & \\
\hline $\begin{array}{l}\text { 3. IC } 3 \text { (É provável que eu compre na } \\
\text { internet) }\end{array}$ & $\begin{array}{c}0,84 \\
(13,14)\end{array}$ & & & \\
\hline
\end{tabular}

Fonte: Elaborada pelos autores.

\subsection{EXAME DAS HIPÓTESES}

Após o exame das medidas utilizadas, o foco deste estudo se volta para a estrutura teórica desenvolvida, que estabelece relações entre os construtos teóricos propostos. A investigação do conjunto de hipóteses foi feita, primariamente, por meio dos índices de ajustamento do modelo estrutural e da significância e 
magnitude dos coeficientes de regressão estimado. Além disso, o coeficiente de determinação foi apresentado, pois representa a proporção de variância da variável dependente que é explicada pelas variáveis independentes.

Os resultados da análise do modelo estrutural, tendo como base o banco de dados com 233 estudantes, encontram-se na Tabela 2. Os índices de ajustamento CFI, NFI, NNFI, todos acima de 0,90 , são considerados satisfatórios. O RMSEA de 0,04 é aceitável. A seguir, consideram-se os coeficientes padronizados de regressão e suas significâncias estatísticas. Tais coeficientes identificam a força das relações causais entre os diversos construtos do modelo, atuando diretamente na confirmação ou rejeição das hipóteses.

No que se refere ao impacto das características pessoais na familiaridade com compras on-line, houve a confirmação da maioria das hipóteses $\left(\mathrm{H}_{\mathrm{I}} \mathrm{A}_{\mathrm{A}}\right.$, $\mathrm{Hio}_{\mathrm{B}}$, Hıо $)$. Apenas o impacto da necessidade de interação sensorial $\left(\mathrm{Hio}_{C}\right)$ não foi confirmado. Dentre as características, a que apresentou maior impacto na familiaridade foi necessidade de interação social $(-0,45)$. A autoeficácia e a inovatividade, apesar do impacto menor, também obtiveram impacto considerável (0,23 e 0,22, respectivamente).

Esses resultados demonstram que as características pessoais impactam diretamente na familiaridade, o que significa que existem relações entre o nível de autoeficácia, inovatividade e necessidade de interação social e o grau de experiência com compras na internet. Consumidores que confiam mais nas suas habilidades com a tecnologia, que buscam mais a inovação e que possuem menor necessidade de um contato pessoal tendem a fazer mais compras na internet, sendo, assim, mais familiares com esse meio de compra virtual. $\mathrm{O}^{2}$, apesar de satisfatório $(0,35)$, indica que outras variáveis, além das estudadas, impactam na familiaridade com compras na internet.

O efeito das características pessoais na atitude ante a compra on-line foi confirmado em parte, pois apenas as necessidades de interações social e sensorial apresentaram efeitos significativos ( $\mathrm{H}_{3}$ e $\left.\mathrm{H}_{5}\right)$. Cabe salientar que o efeito inverso também foi constatado em razão do caráter negativo dessas duas relações. Ambas as variáveis - necessidade de interação sensorial e necessidade de interação social - apresentaram impacto na atitude ante a compra na internet (-o,I2 e -0,I3, respectivamente), impacto esse significativo em nível de 0,05 . 


\section{TABELA 3}

COEFICIENTES DE REGRESSÃO ESTIMADOS AS RELAÇÕES TEÓRICAS ESTABELECIDAS NO MODELO

\begin{tabular}{|c|c|c|c|}
\hline RELACIONAMENTOS DO MODELO & $\begin{array}{c}\text { COEFICIENTE } \\
\text { PADRONIZADO DE } \\
\text { REGRESSÃOa,b }\end{array}$ & HIPÓTESES & $\begin{array}{c}\text { TESTE DE } \\
\text { HIPÓTESES }\end{array}$ \\
\hline \multicolumn{4}{|l|}{ VARIÁVEL DEPENDENTE: FAMILIARIDADE } \\
\hline$R^{2}$ & 0,35 & & \\
\hline Autoeficácia & $0,23(2,99)$ & $\mathrm{H} 10_{\mathrm{A}}$ & Confirmada \\
\hline Necessidade de interação social & $-0,45(-5,17)$ & $\mathrm{H} 10_{\mathrm{B}}$ & Confirmada \\
\hline Necessidade de interação sensorial & $-0,04(-0,49)$ & $\mathrm{H} 10_{\mathrm{C}}$ & Não confirmada \\
\hline Inovatividade & $0,22(2,95)$ & $H 10_{D}$ & Confirmada \\
\hline \multicolumn{4}{|c|}{ VARIÁVEL DEPENDENTE: ATITUDE ANTE A COMPRA PELA INTERNET } \\
\hline$R^{2}$ & 0,70 & & \\
\hline Autoeficácia & $0,04(0,75)$ & $\mathrm{H} 1$ & Não confirmada \\
\hline Necessidade de interação social & $-0,13(-1,94)$ & H3 & Confirmada \\
\hline Necessidade de interação sensorial & $-0,12(-2,32)$ & H5 & Confirmada \\
\hline Inovatividade & $-0,01(-0,20)$ & $\mathrm{H} 7$ & Não confirmada \\
\hline Familiaridade & $0,77(11,91)$ & $\mathrm{H} 12$ & Confirmada \\
\hline \multicolumn{4}{|c|}{ VARIÁVEL DEPENDENTE: INTENÇÃO DE COMPRA PELA INTERNET } \\
\hline$R^{2}$ & 0,61 & & \\
\hline Autoeficácia & $0,05(0,80)$ & $\mathrm{H} 2$ & Não confirmada \\
\hline Necessidade de interação social & $-0,08(-1,11)$ & $\mathrm{H} 4$ & Não confirmada \\
\hline Necessidade de interação sensorial & $-0,07(-1,12)$ & H6 & Não confirmada \\
\hline Inovatividade & $-0,01(-0,19)$ & H8 & Não confirmada \\
\hline Atitude ante a compra pela internet & $0,56(4,73)$ & H9 & Confirmada \\
\hline Familiaridade & $0,17(1,54)$ & $\mathrm{H} 11$ & Não confirmada \\
\hline
\end{tabular}




\section{TABELA 3 (ConClusão)}

\section{COEFICIENTES DE REGRESSÃO ESTIMADOS ÂS RELAÇÕES TEÓRICAS ESTABELECIDAS NO MODELO}

\begin{tabular}{lcc}
\hline RELACIONAMENTOS DO MODELO & $\begin{array}{c}\text { COEFICIENTE } \\
\text { PADRONIZADO DE } \\
\text { REGRESSÃOa, }\end{array}$ & HIPÓTESES \\
\hline ÍNDICES DE AJUSTAMENTO: & $\begin{array}{c}\text { TESTE DE } \\
\text { HIPÓTESES }\end{array}$ \\
\hline$\chi 2$ (Qui-quadrado) & $239,21(p<0,001)$ \\
\hline GL (Graus de liberdade) & 168 \\
\hline CFI (Comparative Fit Index) & 0,97 \\
\hline NFI (Normed Fit Index) & 0,91 \\
\hline NNFI (NonNormed Fit Index) & 0,96 \\
\hline RMR (Root Mean Sq, Residual) & 0,07 \\
\hline $\begin{array}{l}\text { RMSEA (Root Mean Sq, Error of } \\
\text { Approx, }\end{array}$ & 0,044 \\
\hline
\end{tabular}

a As estimativas apresentadas são do software Lisrel.

b $t$-values entre parênteses. Baseado no teste unicaudal: $t$-values $>\mathrm{I}, 65=\mathrm{p}<0,05$; e $t$-values $>2,33=$ $\mathrm{p}<$ ०, oI. Coeficientes significativos em negrito (em nível de o,oI) e em itálico (em nível de ०,05).

Fonte: Elaborada pelos autores.

Também se confirmou, e de maneira bastante significativa, a hipótese I2, que propunha o impacto da familiaridade na atitude $(0,77)$, demonstrando que existe uma relação importante entre experiências anteriores com compras on-line e atitude ante a compra na internet. O coeficiente padronizado de regressão $\left(\mathrm{R}^{2}\right)$ também foi alto $(0,70)$, indicando que as variáveis independentes - características pessoais e familiaridade - conseguiram explicar relativamente bem a variação na atitude ante a compra na internet.

Por fim, analisou-se o impacto de todas as variáveis na intenção do consumidor de comprar futuramente na internet. Nenhuma característica pessoal impactou significativamente nessa variável. Por sua vez, a atitude ante a compra na internet apresentou um impacto bastante alto $(0,56)$, sendo o principal antecedente da intenção de compra dentre as variáveis estudadas. O $\mathrm{R}^{2}$ dessa variável dependente - o,6I - pode ser considerado alto, pelo fato de haver apenas uma 
variável preditora com efeito significativo (atitude), o que pode ser consequência de um certo nível de multicolinearidade dessa última variável com as demais (evidenciada por meio do índice VIF mostrado na Tabela I).

Para entender melhor esses resultados e testar o possível papel de mediação da familiaridade e da atitude ante a compra na internet, foram testados dois modelos alternativos: I. o modelo original sem a presença da familiaridade e 2 . o modelo original sem a atitude. O efeito mediador será diagnosticado se, com a exclusão da variável considerada mediadora, o efeito entre as variáveis independente e dependente analisadas for significativo. Quando se inclui a variável mediadora nessa relação, esse efeito direto decresce significativamente, podendo se tornar insignificante - o que indicaria uma mediação total (BARON; KENNY, I986). Nas tabelas 4 e 5, apresentam-se os resultados encontrados para testar a mediação da atitude e da familiaridade.

Observando a Tabela 4, sem a presença da familiaridade, a autoeficácia passa a exercer um impacto significativo na atitude (0,2I), o mesmo ocorrendo com a inovatividade $(0, I 7)$. A necessidade de interação social, que já exercia um impacto significativo, em nível de ०,05, tem sua magnitude amplificada (de -0,I3 para -0,47, significativo em nível de o,or). Pode-se afirmar, então, que a familiaridade com compras na internet medeia a relação entre as características pessoais e a atitude, com a exceção da necessidade de interação sensorial, o que já era esperado, uma vez que essa variável foi a única que não afetou diretamente na familiaridade. Também, o coeficiente de determinação $\left(\mathrm{R}^{2}\right)$ reduz-se de 0,70 para o,3I, o que indica que a familiaridade explica uma boa parte da variância do construto atitude ante a compra pela internet.

\section{TABELA 4}

\section{MODELO SEM FAMILIARIDADE COMO MEDIADORA}

VARIÁVEL DEPENDENTE:

ATITUDE ANTE A COMPRA PELA INTERNET

\begin{tabular}{lc}
\hline $\mathrm{R}^{2}$ & 0,31 \\
\hline Autoeficácia & $0,21(2,73)^{a, b}$ \\
\hline Necessidade de interação social & $-0,47(-5,30)$ \\
\hline Necessidade de interação sensorial & $-0,09(-1,22)$ \\
\hline Inovatividade & $0,17(2,17)$ \\
\hline
\end{tabular}




\section{TABELA 4 (CONCLUSÃo)}

\section{MODELO SEM FAMILIARIDADE COMO MEDIADORA}

\begin{tabular}{lc} 
VARIÁVEL DEPENDENTE: & \\
INTENÇÃO DE COMPRA & 0,61 \\
\hline$R^{2}$ & $0,06(0,97)$ \\
\hline Autoeficácia & $-0,09(-1,24)$ \\
\hline Necessidade de interação social & $-0,09(-1,43)$ \\
\hline Necessidade de interação sensorial & $0,00(0,01)$ \\
\hline Inovatividade & $0,69(8,17)$ \\
\hline Atitude ante a compra pela internet & $176,78(p<0,001)$ \\
\hline ÍNDICES DE AJUSTAMENTO: & 120 \\
\hline$\chi 2$ (Qui-quadrado) & 0,97 \\
\hline GL (Graus de liberdade) & 0,91 \\
\hline CFI (Comparative Fit Index) & 0,96 \\
\hline NFI (Normed Fit Index) & 0,068 \\
\hline NNFI (NonNormed Fit Index) & 0,046 \\
\hline RMR (Root Mean Sq, Residual) & \\
\hline RMSEA (Root Mean Sq, Error of Approx, & \\
\hline
\end{tabular}

a Coeficientes significativos em negrito (em nível de o,oI) e em itálico (em nível de o,05).

b Coeficiente sublinhados tornaram-se significativos com a retirada da familiaridade do modelo.

Fonte: Elaborada pelos autores.

No que se refere à mediação da atitude na intenção de compras, é possível observar que, quando é retirada a atitude do grupo de variáveis analisadas, a familiaridade, que antes não possuía impacto, passa a ter um impacto bastante significativo (O,6I), o mesmo acontecendo com a necessidade de interação social, que se torna significativa em seu impacto na intenção de compra (de - -,o8, não significativo, para -0,I5, em nível de 0,05 ). Portanto, pode-se dizer que a atitude medeia totalmente o impacto da familiaridade e da necessidade de interação social na intenção de compras na internet. 


\section{TABELA 5}

\section{MODELO SEM ATITUDE COMO MEDIADORA}

\begin{tabular}{|c|c|}
\hline \multicolumn{2}{|l|}{$\begin{array}{l}\text { VARIÁVEL DEPENDENTE: } \\
\text { FAMILIARIDADE }\end{array}$} \\
\hline $\mathrm{R}^{2}$ & 0,35 \\
\hline Autoeficácia & $0,23(3,00)^{a}$ \\
\hline Necessidade de interação social & $-0,45(-5,17)$ \\
\hline Necessidade de interação sensorial & $-0,04(-0,49)$ \\
\hline Inovatividade & $0,22(2,96)$ \\
\hline \multicolumn{2}{|l|}{$\begin{array}{l}\text { VARIÁVEL DEPENDENTE: } \\
\text { INTENÇÃO DE COMPRA }\end{array}$} \\
\hline $\mathrm{R}^{2}$ & 0,52 \\
\hline Autoeficácia & $0,07(1,10)$ \\
\hline Necessidade de interação social & $-0,15(-1,90)^{b}$ \\
\hline Necessidade de interação sensorial & $0,00(-0,01)$ \\
\hline Inovatividade & $-0,02(-0,25)$ \\
\hline Familiaridade & $0,61(7,42)$ \\
\hline \multicolumn{2}{|l|}{ ÍNDICES DE AJUSTAMENTO: } \\
\hline$\chi^{2}$ (Qui-quadrado) & $197,28(p<0,001)$ \\
\hline GL (Graus de liberdade) & 137 \\
\hline CFI (Comparative Fit Index) & 0,97 \\
\hline NFI (Normed Fit Index) & 0,91 \\
\hline RMR (Root Mean Sq, Residual) & 0,071 \\
\hline RMSEA (Root Mean Sq, Error of Approx, ) & 0,045 \\
\hline
\end{tabular}

a Coeficientes significativos em negrito (em nível de 0,05) e em itálico (em nível de 0,05).

b Coeficiente sublinhados tornaram-se significativos com a retirada da atitude do modelo.

Fonte: Elaborada pelos autores. 


\section{CONCLUSÕES}

De modo geral, o modelo desenvolvido parece retratar adequadamente as relações existentes entre as características pessoais, familiaridade, atitude ante a compra na internet e intenção de compra on-line. Os resultados indicam que a atitude ante a compra na internet está no centro da formação do comportamento que impulsionará o consumidor a fazer compras pela internet, e que seu principal antecedente são as experiências anteriores vivenciadas com compras on-line. Ou seja, as predisposições (positivas ou negativas) que o consumidor formou por meio de suas experiências com compras pela internet são o fator decisivo que o levará a comprar na web.

As características pessoais não apresentaram impactos diretos na intenção de compra; todavia, seus efeitos não podem ser desconsiderados, pois eles influenciam o nível de familiaridade do consumidor e, por meio dela, impactam na atitude e na intenção de compra.

O nível de autoeficácia do indivíduo, como esperado, impacta de maneira significativa no nível de familiaridade do consumidor com compras pela internet. Isso demonstra que pessoas que se sentem mais capazes de usar a internet por conta própria, serão de maneira indireta, mais inclinadas a usar a internet para compras, pois terão maior familiaridade e uma atitude mais positiva em relação a esse meio.

Das três características de personalidade que impactaram na familiaridade, a inovatividade foi a que apresentou menor impacto e, quando testada a mediação da familiaridade, ela também teve um impacto pequeno na atitude. Uma possível explicação para esse papel periférico da inovatividade no modelo testado pode ser o fato de que a internet parece não estar sendo mais considerada uma "inovação" por parte do consumidor. Assim, seu impacto no processo de compra on-line estaria se tornando cada vez menor, à medida que a internet passa a fazer parte do dia a dia das pessoas.

A necessidade de interação sensorial, apesar de não exercer nenhuma influência direta na intenção de compra, influencia negativamente a atitude. Isso indica que aqueles indivíduos que sentem maior necessidade de tocar o produto antes da compra terão uma atitude mais negativa ante a internet, o que, por conseguinte, poderá influenciar negativamente sua propensão a comprar na internet. Isso pode ser compreendido, pois a internet ainda não permite esse tipo de contato físico com o produto, o que é valorizado por pessoas com essa característica mais acentuada. Já a variável necessidade de interação social apresentou forte impacto direto na familiaridade e também influência significativa na atitude de compra, destacando-se como uma característica-chave no processo 
de compra on-line. Os resultados demonstraram que pessoas que apreciam o atendimento pessoal serão menos inclinadas ao uso da internet como canal de compra, pois esse meio não privilegia esse tipo de interação, mas sim a tecnologia, como mediadora do processo de compra.

\subsection{IMPLICAÇÕES ACADÊMICAS E GERENCIAIS}

Sob a perspectiva acadêmica, o modelo examina algumas questões relevantes e ainda pouco exploradas no campo de conhecimento do comportamento do consumidor on-line. Conforme Dabholkar e Bagozzi (2002), muitos trabalhos focam aspectos demográficos ou psicográficos do consumidor, mas não se aprofundam em temas mais subjetivos, como o papel da atitude e das características pessoais na formação do comportamento de compra. Sendo assim, este estudo colabora, mesmo que de forma inicial, com o desenvolvimento dessa área, examinando os seguintes temas: I. quais características pessoais se relacionam direta ou indiretamente com o comportamento de compra na internet; 2. quais as relações existentes entre características pessoais e familiaridade; 3 . o papel da atitude na formação do comportamento de compra na web; 4 . a necessidade de interação social como característica-chave no processo de intenção de compra; 5. o teste do papel da mediação da familiaridade entre as características pessoais e a atitude; e 6 . o teste do papel de mediação da atitude entre características pessoais e intenção de compra on-line.

Do ponto de vista gerencial, algumas contribuições podem ser apresentadas. Este estudo possibilita aos gerentes de marketing um entendimento mais adequado sobre os fatores que influenciam a compra eletrônica. O efeito das características pessoais, apesar de não explicar totalmente o que leva um consumidor a comprar na internet, oferece algumas informações que podem melhorar a eficiência do plano de marketing para estimular o uso das lojas virtuais. A variável necessidade de interação social se mostrou fundamental no processo de compra on-line. Isso significa que os consumidores que valorizam o atendimento pessoal não serão compradores tão frequentes das lojas virtuais, a não ser que estas ofereçam mecanismos que supram essa necessidade. Algumas ações como oferecer vendedores on-line disponíveis para suprir dúvidas no momento da compra eletrônica poderiam minimizar o efeito negativo dessa característica. Trabalhar a mensagem de comunicação de marketing, informando que a loja virtual possui vendedores prontos para atender e negociar preços ou simplesmente prestar informações, poderá ajudar a ultrapassar a barreira do atendimento pessoal.

Outro aspecto a ser considerado é o papel da atitude. Considerando que a atitude está no centro da formação do comportamento de compra, é de difícil 
controle e estímulo por parte da empresa, e visto seu caráter subjetivo e a complexidade de sua formação, conforme apresentado neste trabalho, algumas relações identificadas podem ajudar a estimular uma atitude favorável. O papel da familiaridade na formação da atitude demonstra que o custo de uma experiência ruim na internet pode ser bastante alto, prejudicando não apenas a probabilidade de o consumidor retornar a uma loja específica, mas, de forma muito mais significativa, danificando a própria relação que o consumidor estabeleceu com esse meio de compra. Dessa forma, as empresas poderiam investir em mensagens de comunicação institucionais, que promovam o meio da internet como canal de compra e que destaquem sites confiáveis para esse fim.

Ainda, dada a importância da familiaridade na construção das atitudes do consumidor, as empresas devem buscar estratégias para aumentar a familiaridade do internauta com compras em sites. Além disso, sabe-se que, ao se familiarizar com um canal on-line, o consumidor passa a perceber menores riscos nas compras, o que possivelmente o levaria a comprar produtos mais complexos e caros (HOLLOWAY; WANG; PARISH, 2005; SMITH; SIVAKUMAR, 2004). Esse aumento da familiaridade poderia ser gerado por meio de programas de fidelização, em que o cliente acumularia benefícios ao fazer compras no mesmo site, ao longo do tempo, e também pelo desenvolvimento de sites que apresentem maior utilidade e maior facilidade de navegação.

Uma vez que e-consumidores com menos experiência são mais temerosos com relação a aspectos como segurança e privacidade (ANDRADE; KALTCHEVA; WEITZ, 2002), outra maneira de aumentar a confiança e a familiaridade do consumidor com compras pela internet são as certificações digitais oferecidas por muitas empresas e também o desenvolvimento de leis que regulem o comércio eletrônico e deem ao consumidor on-line respaldo para exigir seus direitos em caso de problemas, como fraudes e não entrega de produtos comprados.

\subsection{LIMITAÇÕES E SUGESTÕES DE PESQUISA}

As contribuições trazidas por este estudo devem ser ponderadas pelas limitações que o cercaram. A abordagem de corte transversal e a amostra não probabilística, composta apenas por estudantes de pós-graduação, limitam a generalização dos resultados. Além disso, este estudo abordou somente quatro características pessoais, portanto não se podem fazer afirmações generalizadas sobre a influência das características pessoais nas variáveis dependentes estudadas. Da mesma forma, para medir as atitudes ante a compra pela internet foram utilizados apenas dois itens, enquanto a literatura sobre equações estruturais (HAIR et al., I998) sugere no mínimo três itens medindo cada construto. 
Para pesquisas futuras, sugere-se a inclusão de outras características pessoais, como resistência a mudança, que possam influenciar a intenção de compra on-line. Também, o papel moderador das características pessoais nas relações entre atitudes e intenções de recompra pode ser explorado no futuro, seguindo o trabalho de Dabholkar e Bagozzi (2002). Outra sugestão seria o exame da valência das experiências anteriores, isto é, se as experiências de compra on-line foram positivas ou negativas. Acredita-se que experiências positivas gerarão atitudes ante a compra via internet igualmente favoráveis e vice-versa.

Por fim, sabe-se que alguns produtos são menos padronizados que outros, o que acaba por dificultar a compra desses sem a ocorrência de um contato físico entre consumidor e produto. Por esse motivo, acredita-se que o tipo de produto poderia moderar a relação entre a necessidade de interação sensorial e a intenção de compra, isto é, em compras de produtos mais padronizados, como um livro, o impacto da necessidade de interação sensorial na intenção de compra seria menor do que em compras de produtos menos padronizados, como uma peça de roupa. Sugere-se que esse papel de moderação do tipo de produto seja testado em pesquisas futuras.

\section{THE IMPACT OF PERSONAL TRAITS IN THE INTERNET PURCHASE INTENTION AND THE MEDIATING ROLE OF FAMILIARITY AND ATTITUDE ON ONLINE PURCHASE}

\section{ABSTRACT}

The present research aims to analyze, within the specific context of internet shopping, what is the relationship between personal traits and purchase intention, seeking to understand what is the role of attitude toward online purchase and the consumer familiarity with online shopping in that relationship. A survey was conducted in order to understand the effect of four personal traits: selfefficacy, inherent novelty seeking, need for interaction, and need for touch in the familiarity, in the attitude and in the online purchase intention. Based on the literature review, a model was built and tested, using Confirmatory Factor Analysis to evaluate the measurement model and Structural Equation Modeling (SEM) to test the hypotheses of this work. A total of 233 graduate students answered to the survey. The results demonstrated that there is a significant relationship between personal traits, familiarity and attitude toward online purchase. The need for social interaction was the most relevant personal trait having a direct impact on 
the familiarity and attitude, being a key feature in the online buying process. Furthermore, as a result, it was confirmed that familiarity mediates the relationship between personal traits and attitude toward online shopping. The latter plays a central role shaping the purchasing behavior, being strongly influenced by familiarity, and affecting directly the purchase intention. The theoretical implication of this study is to investigate the still largely unexplored field of knowledge of online consumer behavior, such as the role of attitude and personal traits in the online purchase intention. As a managerial implication, this study gives practitioners a better understanding of the factors that influence online shopping.

\section{KEYWORDS}

Purchase intention; Internet; Personal traits; Familiarity; Attitude.

\section{EL EFECTO DE LAS CARACTERISTICAS PERSONALES EN LA INTENCIÓN DE COMPRA POR INTERNET Y LA MEDIACIÓN DEL PAPEL DE LA FAMILIARIDAD Y DE LA ATITUDE ANTE LA COMPRA POR INTERNET}

\section{RESUMEN}

El presente artículo tiene como objetivo analizar, en el contexto específico de compras por internet, cuál es la relación entre características personales e intención de compra, buscando comprender cuál es el papel de la actitud ante la compra por internet y la familiaridad del consumidor con compras online en esta relación. Una investigación descriptiva conclusiva ha sido elaborada con el propósito de comprender el efecto de cuatro características personales - autoeficacia, innovatividad, necesidad de interacción social y necesidad de interacción sensorial en la familiaridad, en la actitud ante la compra por internet y en la intención de compra online. Con base en la literatura un modelo ha sido construido y testado, utilizándose análisis factorial confirmatorio para valorar el modelo de medidas y análisis de ecuaciones estructurales para testar las hipótesis de este artículo. En total han sido aplicados 233 cuestionarios, y los resultados han indicado que hay una relación significativa entre las características personales estudiadas y la familiaridad con las compras por internet. La necesidad de interacción social ha impactado directamente la familiaridad y la actitud, destacándose como una característica esencial en el proceso de compra online. Además, como resultado, 
se ha confirmado que la familiaridad media las relaciones entre las características personales y la actitud ante la compra online. Esta juega un rol central en la formación del comportamiento de compra, siendo fuertemente influenciada por la familiaridad e influenciando directamente las intenciones de compra. La implicación teórica de este artículo es examinar cuestiones aún poco exploradas en el área de conocimiento del comportamiento del consumidor online como actitud y el papel de las características personales en la intención de compra online. Como implicación gerencial, este artículo ofrece a los administradores más entendimiento sobre los factores que influencian las compras electrónicas.

\section{PALABRAS CLAVE}

Intención de compra; Internet; Características personales; Familiaridad; Actitud.

\section{REFERÊNCIAS}

AGARWAL, R.; PRASAD, J. A conceptual and operational definition of personal innovativeness in the domain of information technology. Information Systems Research, Hanover, v. 9, n. 2, p. 2042I5, June I998.

ANDRADE, E. B.; KALTCHEVA, V.; WEITZ, B. Self-disclosure on the web: the impact of privacy policy, reward, and company reputation. Advances in Consumer Research, Valdosta, v. 29, n. I, p. 350-353, Jan. 2002.

BANDURA, A. Self-efficacy mechanism in human agency. American Psychologist, Washington, v. 37, n. 2, p. I22-I47, Feb. I982.

. Social cognitive theory of personality. In: PERVIN, L. (Org.). Handbook of personality theory and research. New York: Guilford, I999. p. 154-196.

BARON, R. M.; KENNY, D. A. The moderator-mediator variable distinction in social psychological research: conceptual, strategic, and statistical considerations. Journal of Personality and Social Psychology, Storrs, v. 5I, n. 6, p. II73-II82, Dec. I986.

BART, Y. et al. Are the drivers and role of online trust the same for all websites and consumers? A large-scale exploratory empirical study. Journal of Marketing, Chicago, v. 69, n. 4, p. I33-I52, Oct. 2005.

BELLMAN, S.; LOHSE, G. L.; JOHNSON, E. J. Predictors of online buying behavior. Communications of the ACM, New York, v. 42, p. 32-38, Dec. I999.

BROWN, M.; POPE, N.; VOGES, K. Buying or browsing? An exploration of shopping orientations and online purchase intention. European Journal of Marketing, West Yorkshire, v. 37, n. II/I2, p. I666-I684, Dec. 2003.

BRUNER II, J. C.; KUMAR, G. C. Web commercials and adverstising hierarchy-of-effects. Journal of Adverstising Research, New York, v. 40, p. 35-42, Nov. 2000.

CAPRARA, G. V.; CERVONE, D. Personality: determinants, dynamics, and potentials. New York: Cambridge University Press, 2000. 
CHEN, G.; GULLY, S. M.; EDEN, D. Validation of a new general self-efficacy scale. Organizational Research Methods, California, v. 4, n. I, p. 62-83, Jan. 2001.

CHURCHILL, G. Marketing research. Orlando: Fryden Press, I999.

CLARKE III, I.; FLAHERTY, T. B. Advances in eletronic marketing. Hershey: IGI Publishing. 2005. DABHOLKAR, P. A. Consumer evaluations of new technology-based self-service options: an investigations of alternative models of service quality. Journal of Research in Marketing, Amsterdam, v. I3, n. I, p. 29-5I, Feb. I996.

DABHOLKAR, P. A.; BAGOZZI, R. P. An attitudinal model of technology based self-service. Moderating effects of consumer traits and situational factors. Journal of Academy of Marketing Science, Thousand Oaks, v. 30, n. 3, p. I84-201, June 2002.

DAVIS, F. D.; BAGOZZI, R. P.; WARSHAW, P. R. User acceptance of computer technology: a comparison of two theoritical models. Management Science, Hanover, v. 35, n. 8, p. 982, Aug. 1989. EBIT-EMPRESA. WebShoppers $23^{a}$ edição. Disponível em: <http://www.e-bit.com.br>. Acesso em: 6 jun. $201 \mathrm{I}$.

ENGEL, J. F.; BLACKWELL, R. D.; MINIARD, P. W. Comportamento do consumidor. 8. ed. Rio de Janeiro: LTC, 2005.

FALK, H.; TALARZYK, W.; WIDING II, R. E. Retailing and online consumer information services. International Journal of Retail and Distribution Management, Germantown, v. 22, n. 7, p. I8-23, Nov. I994.

GOLDSMITH, R. E.; BRIDGES, E. E-tailing vs. retailing. Using attitudes to predict online buying behavior. Quarterly Journal of Electronic Commerce, Hershey, v. I, n. 3, p. 245-253, 2000.

HAIR, J. et al. Multivariate data analysis. New Jersey: Prentice Hall, I998.

HAUSMAN, A.; SIEKPE, J. The effect of web interface features on consumer online purchase intentions. Journal of Business Research, New York, v. 62, n. I, p. 5-I3, Jan. 2009.

HIRSCHMAN, E. C. Innovativeness, novelty seeking and consumer creativity. Journal of Consumer Research, Mahloah, v. 7, n. 3, p. 283-295, Dec. 1980.

HOLLOWAY, B.; WANG, S.; PARISH, J. The role of cumulative online purchasing experience in service recovery management. Journal of Interactive Marketing, New York, v. I9, n. 3, p. 54-66, Aug. 2005 .

KIM, J.; LEE, H. C.; KIM, H. J. Factors affecting online search intention and online purchase intention. Seoul Journal of Business, v. IO, n. 2, p. 27-47, Dec. 2004.

KULVIWAT, S.; BRUNER II, J. C.; NEELANKAVIL, J. P. The role of self-efficacy in predicting technology acceptance. Hempstead: Hofstra University, Frank G. Zarb School of Business, Department of Marketing and International Business, 2005. 30p. Working paper ZSB 040501. Disponível em: <http:// www.hofstra.edu/pdf/BIZ_mlc_workingpaperıı.pdf>. Acesso em: 7 jul. 2009 .

LAROSE, R.; EASTIN, M. S Internet self-efficacy and the psychology of the digital divide. Journal of Computer-Mediated Communication, Malden, v. 6, Sept. 2000.

LEE, K. S.; TAN, S. J. E-retailing versus physical retailing: a theoretical model and empirical test of consumer choice. Journal of Business Research, New York, v. 56, n. II, p. 877-886, Nov. 2005.

MEHRABIAN, A.; RUSSEL, J. A. An approach to environmental psychology. Cambridge: MIT Press, I974.

MEUTER, M. L. et al. Self-service technologies: understanding customer satisfaction with technology-based service encounters. Journal of Marketing, Chicago, v. 64, n. 3, p. 50-64, July 2000. 
NETEMEYER, R. G; BEARDEN, W. O.; SHARMA, S. Scaling procedures: issues and applications. Thousand Oaks: Sage, 2003.

NOVAK, T. P.; HOFFMAN, D. L.; YUNG, Y. Measuring the customer experience in online environments: a structural modeling approach. Marketing Science, Linthicum Heights, v. I9, n. I, p. 22-42. Nov./Dec. 2000.

O'BRIEN, R. A caution regarding rules of thumb for variance inflation factors. Quality and Quantity, Kountze, v. 4I, n. 5, p. 673-690, Oct. 2007.

PECK, J.; CHILDERS, T. L. Individual differences in haptic information processing: the "Need for Touch" Scale. Journal of Consumer Research, Chicago, v. 30, n. 3, p. 430-442, Dec. 2003.

ROGERS, E. M. Diffusion of innovations. 4. ed. New York: The Free Press, I995.

SANTOS, C. P.; FERNANDES, D. V. H. Failure and recovery in internet purchasing: their impact on consumer trust and loyalty to the company's site and to the online shopping environment. In: ENANPAD, 3I., 2007, Rio de Janeiro. Anais... Rio de Janeiro: Anpad, 2007. p. I-I6.

SHETH, J. N.; MITTAL, B.; NEWMAN, B. I. Comportamento do cliente: indo além do comportamento do consumidor. São Paulo: Atlas, 200I.

SIMON, F.; USUNIER, J.-C. Usunier. Situational determinants of service customer preference for personnel-in-contact over self-service technology. International Journal of Research in Marketing, v. 24, n. 2, p. I63-I73, July 2007.

SMITH, D.; SIVAKUMAR, K. Flow and Internet shopping behavior: a conceptual model and research propositions. Journal of Business Research, New York, v. 57, n. IO, p. II99-I208, Oct. 2004. STAJKOVIC, A. D.; LUTHANS, F. Self-efficacy and work-related performance: a meta-analysis. Psychological Bulletin, Washington, v. I24, n. 2, p. 240-26I, Sept. I998.

TIPTON, R. M.; WORTHINGTON, E. L. The measurement of generalized self-efficacy: a study of construct validity. Journal of Personality Assessment, Oxfordshire, v. 48, n. 2, p. 545-48, Sept. I984. YEO, G.; NEAL, A. An examination of the dynamic relationship between self-efficacy and performance across levels of analysis and levels of specificity. Journal of Applied Psychology, Washington, v. 9I, n. 5, p. Iо8-IOI, Oct. 2006.

YOH, E. et al. Consumer adoption of the internet: the case of apparel shopping. Psychology \& Marketing, Malden, v. 20, n. I2, p. I095-III8, Dec. 2003.

YOON, S. The antecedents and consequences of trust in online-purchase decisions. Journal of Interactive Marketing, New York, v. I6, n. 2, p. 47-63, May 2003.

ZHAO, F. Predicting online customer shopping behavior. In: KHOSROW-POUR, M. Emerging trends and challenges in information technology. Washington: Idea Group Pub, 2006. p. 846-849. ZHOU, L. et al. Online shopping acceptance model: a critical survey of consumer factors in online shopping. Journal of Electronic Commerce, Chico, v. 8, n. I, p. 4I-62, Feb. 2007. 